\title{
Hepatitis B virus-specific T cells associate with viral control upon nucleos(t)ide-analogue therapy discontinuation
}

\author{
Laura Rivino, ${ }^{1}$ Nina Le Bert, ${ }^{1,2}$ Upkar S. Gill, ${ }^{1,3}$ Kamini Kunasegaran, ${ }^{1}$ Yang Cheng, ${ }^{4}$ Damien Z.M. Tan, ${ }^{2}$ Etienne Becht, ${ }^{4}$ \\ Navjyot K. Hansi, ${ }^{3}$ Graham R. Foster, ${ }^{3}$ Tung-Hung Su, ${ }^{5}$ Tai-Chung Tseng, ${ }^{5}$ Seng Gee Lim, ${ }^{6}$ Jia-Horng Kao, ${ }^{5}$ Evan W. Newell, ${ }^{4}$ \\ Patrick T.F. Kennedy, ${ }^{3}$ and Antonio Bertoletti ${ }^{1,2,4}$

\begin{abstract}
'Emerging Infectious Diseases Programme, Duke-NUS Medical School, Singapore. ${ }^{2}$ Infection and Immunity Program, Singapore Institute for Clinical Sciences, Agency for Science, Technology and Research (A*STAR), Singapore. ${ }^{3}$ Hepatology, Centre for Immunobiology, Blizard Institute, Barts and The London School of Medicine and Dentistry, Queen Mary University of London (QMUL), London, United Kingdom (UK). ${ }^{4}$ Singapore Immunology Network, A*STAR, Singapore. ${ }^{5}$ Division of Gastroenterology, Department of Internal Medicine, National Taiwan University Hospital, Taipei, Taiwan. ${ }^{6}$ Division of Gastroenterology and Hepatology, Department of Medicine, National University Health System, Singapore.
\end{abstract}

BACKGROUND. The clinical management of chronic hepatitis B virus (HBV) patients is based exclusively on virological parameters that cannot independently determine in which patients nucleos(t)ide-analogue (NUC) therapy can be safely discontinued. NUCs efficiently suppress viral replication, but do not eliminate HBV. Thus, therapy discontinuation can be associated with virological and biochemical relapse and, consequently, therapy in the majority is life-long.

METHODS. Since antiviral immunity is pivotal for HBV control, we investigated potential biomarkers for the safe discontinuation of NUCs within immune profiles of chronic HBV patients by utilizing traditional immunological assays (ELISPOT, flow cytometry) in conjunction with analyses of global non-antigen-specific immune populations (NanoString and (yTOF). Two distinct cohorts of 19 and 27 chronic HBV patients, respectively, were analyzed longitudinally prior to and after discontinuation of 2 different NUC therapy strategies.

RESULTS. Absence of hepatic flares following discontinuation of NUC treatment correlated with the presence, during NUC viral suppression, of HBV core and polymerase-specific T cells that were contained within the ex vivo PD-1+ population.

CONCLUSIONS. This study identifies the presence of functional HBV-specific T cells as a candidate immunological biomarker for safe therapy discontinuation in chronic HBV patients. Furthermore, the persistent and functional antiviral activity of PD-1+ HBV-specific T cells highlights the potential beneficial role of the expression of $\mathrm{T}$ cell exhaustion markers during human chronic viral infection.

FUNDING. This work was funded by a Singapore Translational Research Investigator Award (NMRC/STaR/013/2012), the Eradication of HBV TCR Program (NMRC/TCR/014-NUHS/2015), the Singapore Immunology Network, the Wellcome Trust (107389/Z/15/Z), and a Barts and The London Charity (723/1795) grant.

Related Commentary: p. 573

Authorship note: L. Rivino and N. Le Bert contributed equally to this work. P.T.F. Kennedy and $A$. Bertoletti contributed equally to this work.

Conflict of interest: A. Bertoletti declares the following relationship with commercial entities developing therapeutics for HBV treatment. He collaborates and receives research support from Gilead Sciences Inc. to test the effect of HBV antigens on immune cell function. He acted as a consultant and served on the advisory boards of Gilead Sciences Inc., Medlmmune, Janssen-Cilag, IONIS Pharmaceuticals, Abivax, and HUMABS BioMed. He is also a cofounder of Lion TCR, a biotech company developing T cell receptors for treatment of virus-related cancers and chronic viral diseases. P.T.F. Kennedy has collaborative grant funding from Gilead Sciences Inc., participates in advisory board/provides consultancy to Gilead Sciences Inc. and Janssen, and is an investigator for industry-led trials with Gilead Sciences Inc., Janssen, Alere, and Assembly Biosciences.

Submitted: January 13, 2017; Accepted: November 7, 2017.

Reference information: / Clin Invest. 2018;128(2):668-681.

https://doi.org/10.1172/JCI92812

\section{Introduction}

Hepatitis B virus (HBV) remains a major global healthcare challenge, with over 400 million chronically infected worldwide (1). HBV is a DNA virus that infects hepatocytes and establishes a mini-chromosome (cccDNA) that acts as a template for HBV replication and antigenic production (2). Nucleos(t)ide-analogue (NUC) therapy suppresses viral replication, but does not successfully eliminate cccDNA, thus leading to HBV rebound and development of hepatic flares upon treatment withdrawal (3). Life-long NUC therapy, which is therefore required in the majority, represents a financial burden for patients and national health systems. Concerns of potential drug toxicity (4) and the selection of mutants that could possibly escape prophylactic vaccination are also important considerations (5). Thus, a major unmet need in the management of chronic HBV patients is the definition of bio- 


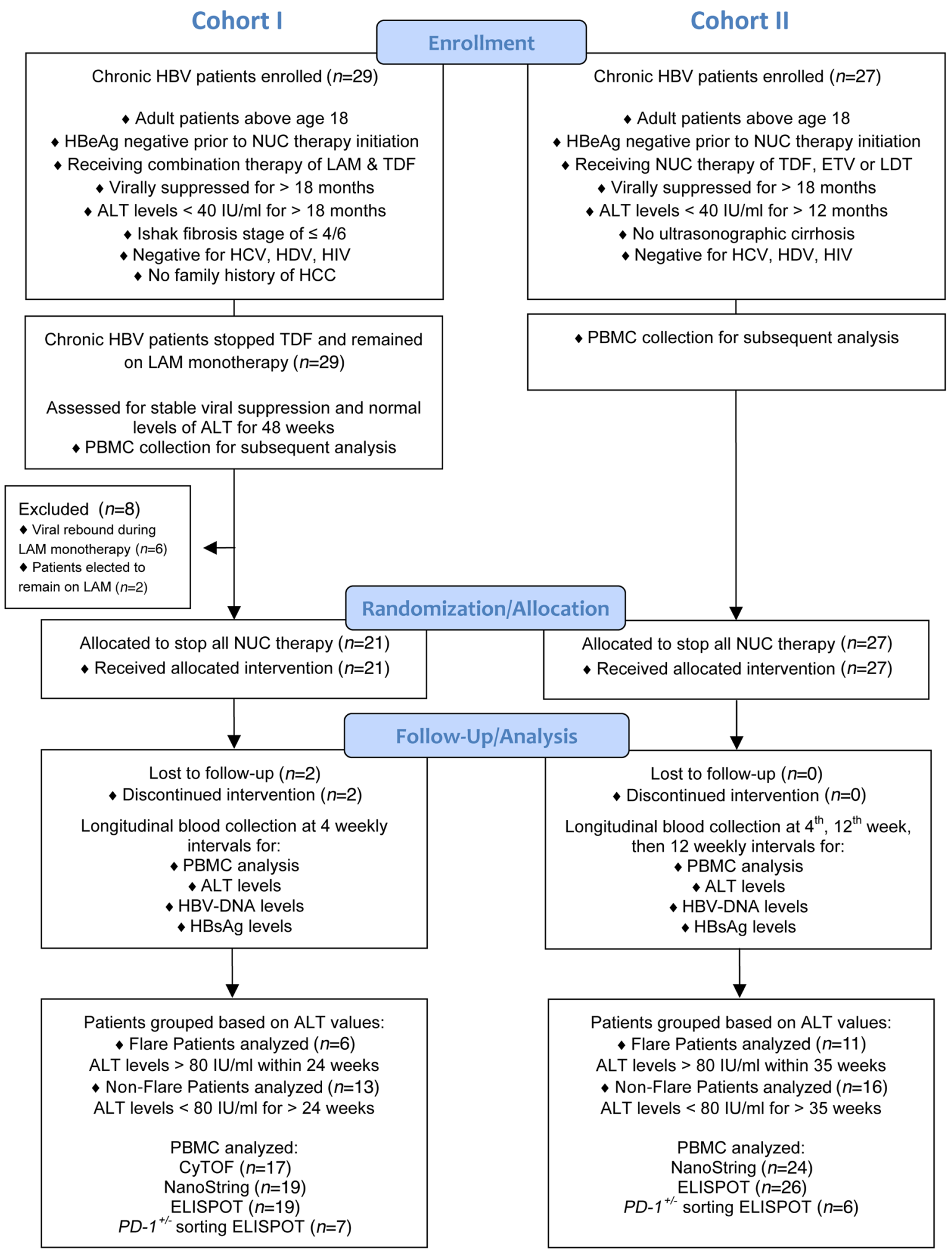

Figure 1. CONSORT-style flow diagram. Enrollment, follow-up, and analysis before and after stopping NUC therapy for the UK (cohort 1, left) and Asian (cohort 2, right) chronic HBV patient cohorts. For more details on the enrolled patients, refer to Tables 1 and 2. HCC, hepatocellular carcinoma.

markers that can predict the safe discontinuation of NUC therapy. There is no consensus on current treatment guidelines on the optimal time to consider stopping NUC therapy (6). Seroconversion of the surface antigen of HBV (HBsAg) or HBsAg to values below 100
$\mathrm{IU} / \mathrm{ml}$ (7) in HBV e-antigen-negative (HBeAg-negative) patients is recommended by some as a safe stopping point; however, such values are observed in a minority of NUC-treated patients. The recent FINITE study of stopping NUC therapy in HBeAg patients 
A

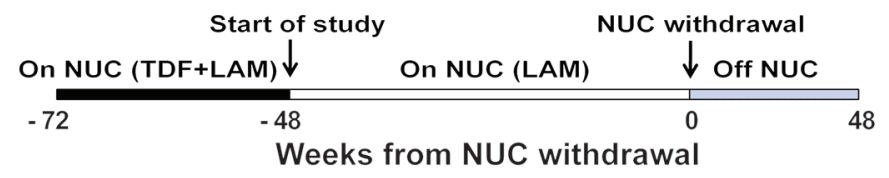

B

B

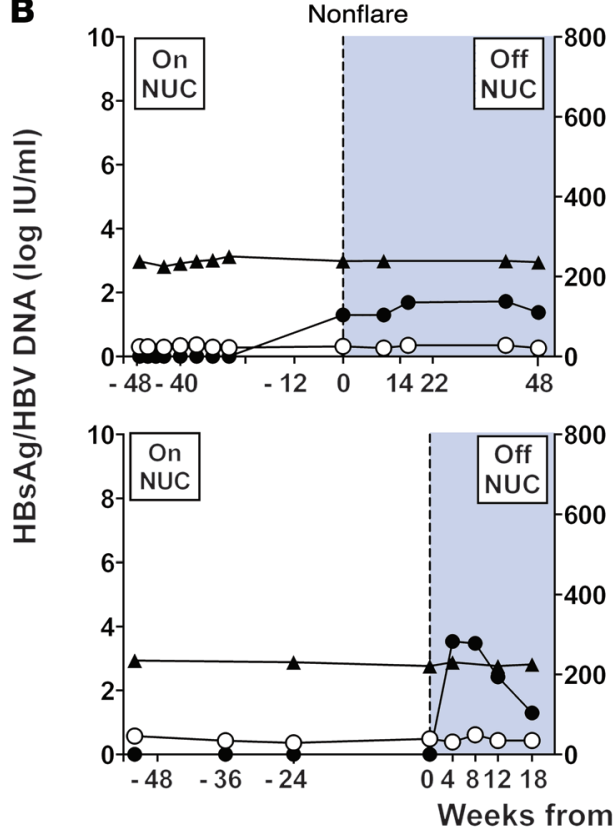

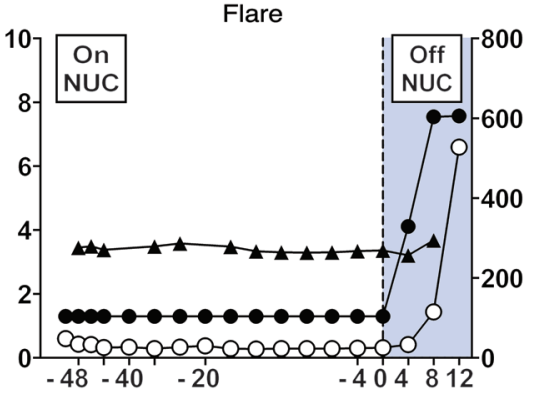

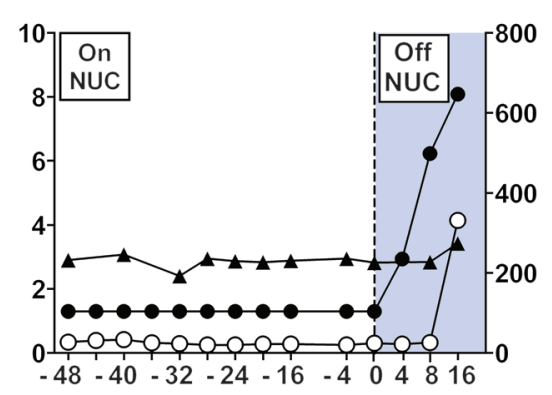

- HBsAg

- HBV DNA

O ALT

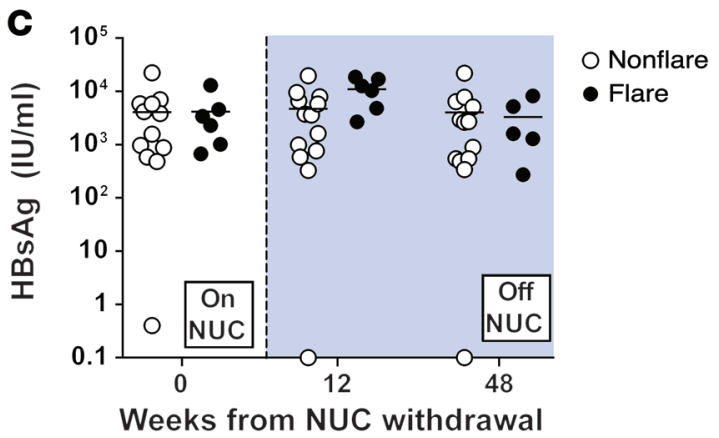

Figure 2. Characteristics of the patient cohort. (A) Schematic depicting the study design. Patients were enrolled for the study and maintained on NUC therapy for 1 year (48 weeks), after which therapy was discontinued. Weeks are calculated from therapy discontinuation (week 0 ). (B) Upon stopping therapy, patients were classified as nonflare ( 2 representative patients [ $n=13]$, left top and bottom panels) or flare (2 representative patients [ $n=6]$, right top and bottom panels) based on ALT and HBV DNA values observed in the 6 months ( 24 weeks) following therapy discontinuation. Nonflare and flare, patients with ALT values below or above $2 \times$ ULN ( $80 \mathrm{IU} / \mathrm{ml})$, respectively. (C) Serum HBsAg values observed in all patients, subdivided as flare and nonflare upon therapy withdrawal. HBsAg values are measured longitudinally at weeks 0,12 , and 48 from NUC withdrawal. HBsAg values are expressed as IU/ml.

reports that 13 out of 21 patients were able to remain off therapy for nearly 3 years, yet no criteria that distinguishes which patients can safely discontinue therapy is identified (8). Since antiviral immunity plays a critical role in the suppression and control of HBV infection, we sought to identify immunological biomarkers to predict whether NUC therapy can be safely discontinued.

During the natural history of chronic HBV, the quantity and function of HBV-specific T cells are shown to correlate with $\mathrm{HBV}$ control (9). Moreover, components of innate immunity, such as NK cells, can modulate HBV control or HBV-specific T cell quantity (10). Recent studies show that NK cells with a less "inflammatory" phenotype (expressing lower frequencies of CD38, Ki-67, and TRAIL) were associated with HBsAg seroconversion and may represent a potential marker for safe NUC suspension (11). Furthermore, it was recently reported that a transcriptional $\mathrm{T}$ cell signature that is linked to $\mathrm{CD} 8^{+} \mathrm{T}$ cell exhaustion and $\mathrm{CD} 4^{+} \mathrm{T}$ cell costimulation can predict the outcome of chronic viral infection (12).

Thus, in order to define possible immune markers associated with HBV control or viral relapse upon NUC discontinuation, we characterized the functional profile of both antigen-specific $\mathrm{T}$ cells and global non-antigen-specific immunity in chronic HBV patients during NUC therapy. Traditional immunological assays (ELISPOT, flow cytometry) (13) were utilized to detect the frequency, functionality, and fine specificity of HBV-specific T cells in conjunction with methods of analysis of the global non-antigen-specific immune populations (Cytometry by Time-of-Flight [CyTOF], NanoString). CyTOF, a mass cytometry technology (14), was used to define the frequency and phenotype of global T, NK, and B cell populations, while NanoString technology was utilized to analyze the transcriptional profiles of immune gene expression in purified $\mathrm{CD} 4^{+}$and $\mathrm{CD} 8^{+} \mathrm{T}$ cell subsets.

Two distinct cohorts of 19 and $27 \mathrm{HBeAg}$-negative chronic HBV patients who withdrew from long-term antiviral therapy were studied longitudinally during and after stopping NUC treatment. Results from the 2 cohorts demonstrate that patients who discontinue NUC therapy without subsequently developing hepatic flares are characterized by increased frequencies of HBV-specific T cells, which can be expanded preferentially from the PD $-1^{+} \mathrm{T}$ cell 
Table 1. Details of the therapy discontinuation cohort of chronic HBV patients (cohort 1)

\begin{tabular}{|c|c|c|c|c|c|c|c|c|c|c|c|}
\hline & \multicolumn{4}{|c|}{ Patient details } & \multicolumn{2}{|c|}{ Before NUC } & \multirow{2}{*}{$\begin{array}{c}\text { At stop NUC } \\
\text { HBsAg } \\
\text { (IU/ml) }\end{array}$} & \multicolumn{4}{|c|}{ After NUC } \\
\hline & ID & Sex & $\begin{array}{c}\text { Age at } \\
\text { enrollment (yr) }\end{array}$ & $\begin{array}{c}\text { HBV } \\
\text { genotype }\end{array}$ & $\begin{array}{l}\text { ALT } \\
\text { (IU/I) }\end{array}$ & $\begin{array}{c}\text { HBV DNA } \\
\left(10^{3} \mathrm{IU} / \mathrm{ml}\right)\end{array}$ & & $\begin{array}{l}\text { Peak ALT } \\
\text { (IU/I) (wk) }\end{array}$ & $\begin{array}{c}\text { Max HBV DNA } \\
\left(10^{3} \mathrm{IU} / \mathrm{ml}\right)\end{array}$ & $\begin{array}{l}\log _{2} \text { fold-change } \\
\text { HBV DNA after NUC }\end{array}$ & $\begin{array}{c}\text { Back on } \\
\text { NUC (wk) }\end{array}$ \\
\hline \multicolumn{12}{|l|}{ Nonflare } \\
\hline & NF 2 & M & 60 & D & NA & NA & 0.4 & $13(0)$ & 0.02 & NA & № (>255) \\
\hline & NF 3 & M & 49 & C & 79 & 213.80 & 585 & $49(8)$ & 3.45 & -5.95 & № $(>296)$ \\
\hline & NF 4 & $\mathrm{~F}$ & 32 & D & 113 & 758.58 & 6964 & $80(24)$ & 29.35 & -4.69 & Yes (82) \\
\hline & NF 6 & M & 55 & D & 47 & 40738.03 & 5795 & $80(48)$ & 64.56 & -9.30 & Yes (48) \\
\hline & NF 7 & $\mathrm{~F}$ & 65 & D & 52 & 1023.29 & 5826 & $73(16)$ & 654.08 & -0.65 & Yes (16) \\
\hline & NF 8 & M & 52 & D & 87 & 3235.94 & ND & $79(56)$ & 0.17 & -14.20 & Yes (60) \\
\hline & NF 9 & M & 38 & D & 30 & 1.23 & 1573 & $66(8)$ & 12.37 & 3.33 & No $(>220)$ \\
\hline & NF 10 & M & 50 & B & 28 & 165.96 & 481 & $67(10)$ & 172.10 & 0.05 & Yes (46) \\
\hline & NF 11 & $\mathrm{~F}$ & 44 & A & 46 & 21.38 & 4152 & $30(8)$ & 255.67 & 3.58 & Yes (84) \\
\hline & $\mathrm{F} 1$ & M & 47 & D & 131 & 14.79 & 4576 & $209(8)$ & $53 \times 10^{3}$ & 11.81 & Yes (8) \\
\hline & $\mathrm{F} 2$ & M & 40 & D & 133 & 4677.35 & 13046 & $154(24)$ & $22.4 \times 10^{3}$ & 2.26 & Yes (24) \\
\hline & F3 & M & 62 & C & 60 & 3890.45 & 672 & $332(12)$ & $122.2 \times 10^{3}$ & 4.97 & Yes (12) \\
\hline & $\mathrm{F} 4$ & $M$ & 50 & B & 58 & 407.38 & 3389 & $197(12)$ & $337.6 \times 10^{3}$ & 9.69 & Yes (12) \\
\hline & F 5 & M & 44 & $D$ & 49 & 1.02 & 2293 & $528(12)$ & $37.3 \times 10^{3}$ & 15.15 & Yes (12) \\
\hline & F 6 & M & 43 & $D$ & NA & NA & 1018 & $565(16)$ & $1200 \times 10^{3}$ & NA & Yes (8) \\
\hline
\end{tabular}

fraction. Thus, this study proposes a candidate biomarker for safe therapy withdrawal and, in addition, suggests that during human chronic viral infection, virus-specific T cells expressing inhibitory markers may escape deletion and retain a partial functional capacity that allows them to mediate protective antiviral immunity.

\section{Results}

Definition of the chronic HBV patient cohort. Twenty-nine $\mathrm{HBeAg}$-negative chronic HBV patients on long-term antiviral therapy with a combination of tenofovir disoproxil fumarate (TDF) and lamivudine (LAM) (virally suppressed with normal levels of transaminases for at least 18 months) were enrolled (Figure 1, cohort 1). At the start of the study, for safety concerns, one drug was discontinued first (TDF), after which patients received antiviral monotherapy with LAM for 48 weeks before complete NUC withdrawal. Patients were routinely monitored at 4-week intervals, and peripheral blood was longitudinally sampled during the LAM monotherapy and following complete therapy discontinuation for up to 2 years (Figure 2A). During the 48 weeks of monotherapy, 6 of the enrolled patients experienced an increase in HBV DNA and were excluded and another 2 patients elected to remain on LAM monotherapy. Following complete NUC discontinuation, patients differentiated into 2 groups based on the presence or absence of hepatic flares during a 6-month (24 week) period subsequent to treatment withdrawal. Nonflare or flare patients were defined based on serum alanine aminotransferase (ALT) values below $(\leq 80 \mathrm{IU} / \mathrm{ml}$ ) or above $(>80 \mathrm{IU} / \mathrm{ml}) 2 \times$ the upper limit of normal (ULN), respectively. During viral rebound, patients with hepatic flares $(n=6 / 19=31.6 \%)$ displayed levels of HBV DNA that were 100 times greater compared with those that did not flare $(n=$ $13 / 19=68.4 \%$ ) (Figure 2B and Table 1 ). HBsAg values during NUC therapy and HBV DNA levels before the start of therapy did not differ in patients from the 2 groups (Figure $2 \mathrm{C}$ and Table 1 ).

Increased frequencies of $\mathrm{HBV}$-specific $\mathrm{T}$ cells in patients with $\mathrm{HBV}$ control. During the natural history of HBV, viral control is proportionally associated with greater frequencies of $\mathrm{HBV}$-specific $\mathrm{T}$ cells (15), which are also critical for viral control in a nonhuman primate model (16). We thus asked whether the HBV-specific $\mathrm{T}$ cell response differs between those who develop viral relapse and those who do not upon NUC withdrawal. In line with previous studies reporting the inability to detect HBV-specific T cells ex vivo in chronic HBV patients $(13,15,17)$, we were unable to detect HBV-specific $\mathrm{T}$ cells in the peripheral blood of $\mathrm{HLA}-\mathrm{A}^{*} 0201^{+}$patients by using HBV-peptide HLA-A*0201 pentamers (Supplemental Figure 1A; supplemental material available online with this article; https://doi.org/10.1172/JCI92812DS1). To increase the sensitivity of detection of HBV-specific T cells and comprehensively test the presence of $\mathrm{T}$ cells specific for the whole HBV proteome, we expanded patient peripheral blood mononuclear cells (PBMCs) for 10 days with a panel of overlapping 15-mer peptides spanning the HBV proteome ( $x$, core, envelope, and polymerase), as described previously (13). $T$ cell recognition of the peptides pooled according to the single proteins was subsequently tested by IFN- $\gamma$ ELISPOT. Patient PBMCs were tested at 3 time points prior to and after stopping therapy (weeks $-36,-12,+28$, where week 0 represents the time of therapy discontinuation). We show that the frequencies of in vitro-expanded HBV-specific T cells during NUC therapy were significantly higher in patients that did not flare upon therapy with- 
A

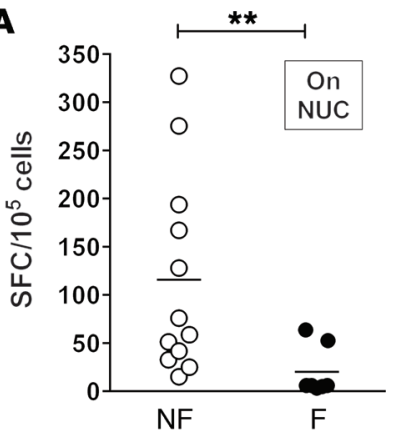

B

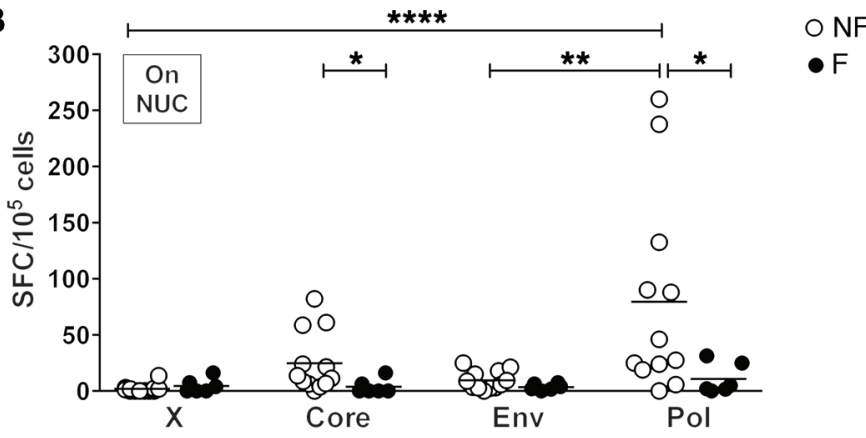

$\mathrm{NF}$

$\mathrm{F}$
C

$c$

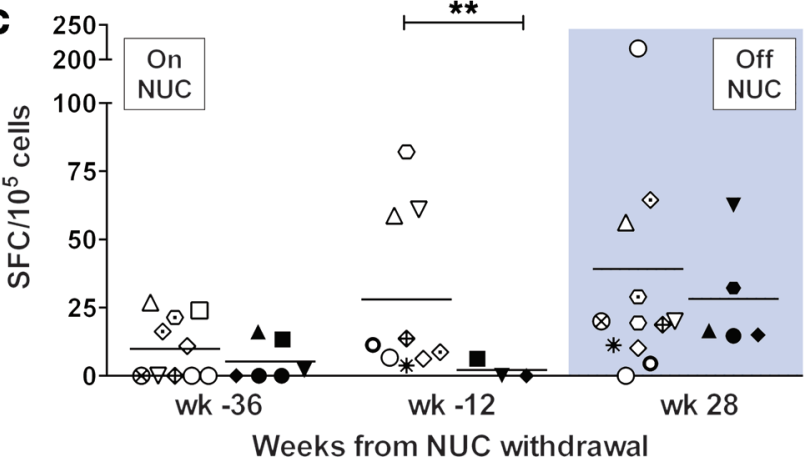

E

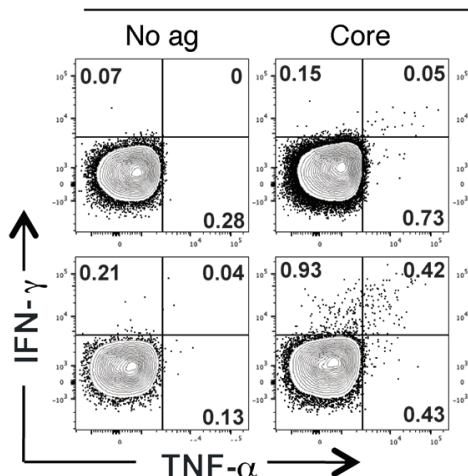

$\mathbf{F}$

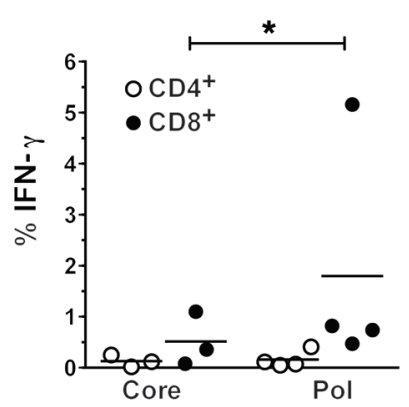

$\mathrm{NF}$
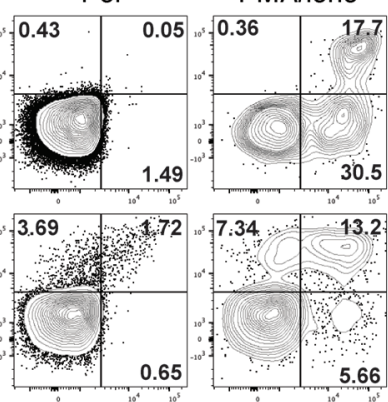

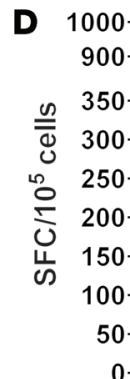

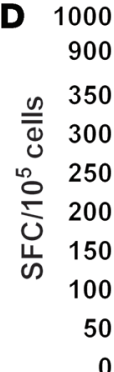


A
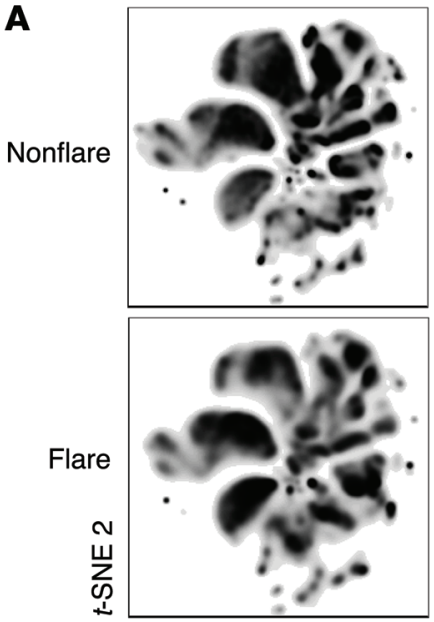

t-SNE 1
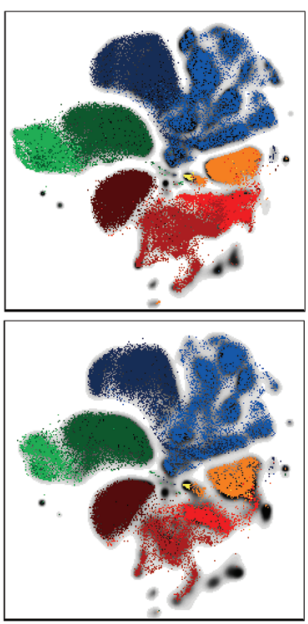

B
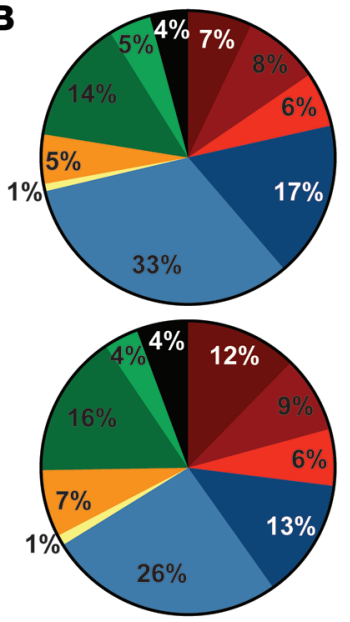

Naive CD8 ${ }^{+} \mathrm{T}$

Memory CD8 ${ }^{+} \mathrm{T}$

$\mathrm{T}_{\mathrm{EMRA}} \mathrm{CD} 8^{+} \mathrm{T}$

Naive $C D 4^{+} T$

Memory CD4 ${ }^{+} \mathrm{T}$

CD56 bright $\mathrm{NK}$

CD56 ${ }^{\text {dim }} \mathrm{NK}$

Naive B

Memory B

Other
C

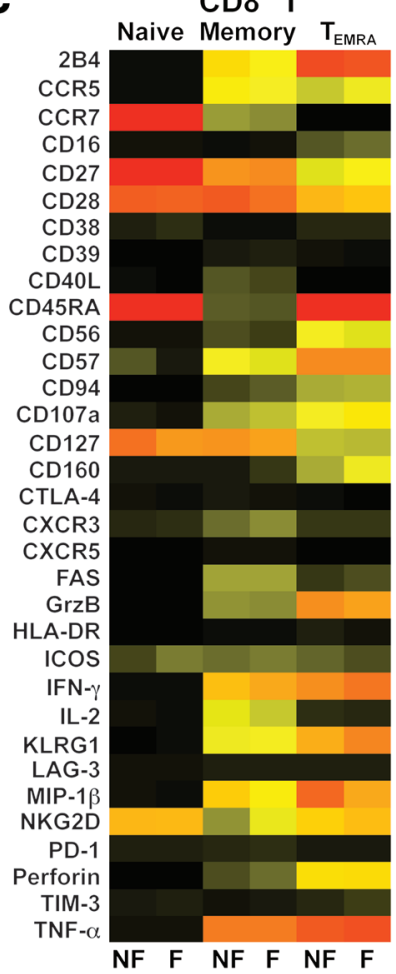

$\mathrm{CD}^{+} \mathrm{T}$ Naive Memory

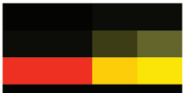

\section{西}
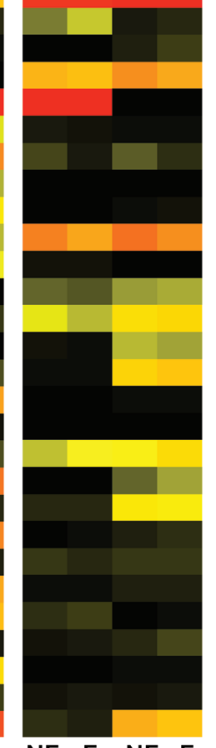

NF $F$ NF F
NK

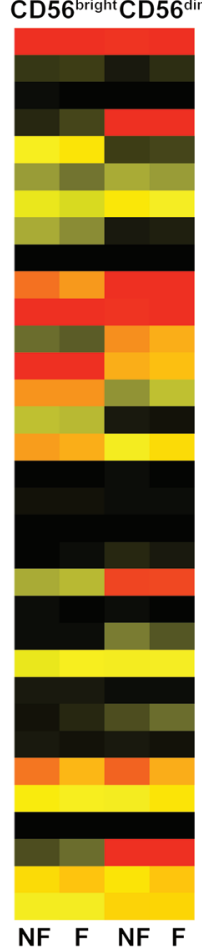

B

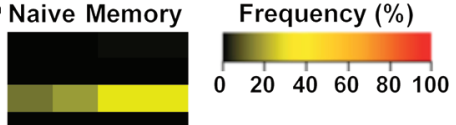

Figure 4. High-dimensional analyses by CyTOF of immune populations present in the peripheral blood of patients with and without evidence of flares upon therapy discontinuation. PBMCs from patients with and without flares upon therapy discontinuation were briefly stimulated with PMA/ionomycin, stained with a panel of 40 antibodies, and analyzed by CyTOF. (A) Live cells were concatenated after downsampling and analyzed in parallel by $t$-SNE (left panels). Manually gated lymphocyte populations were then overlaid onto the total $t$-SNE map (right panels). (B) Pie charts showing the average relative frequency of the different lymphocyte populations within the 2 groups of patients. (C) Heatmaps showing the average frequency of expression of each immunological marker within the indicated lymphocyte populations of patients that flared or did not flare upon therapy discontinuation. The percentages of positive expression are shown from low (black) to high (red). $t$-SNE plot shown in $\mathbf{A}$ shows 1 representative experiment. Data shown in $\mathbf{B}$ and $\mathbf{C}$ represent the average expression from 17 patients ( $n=12$ nonflare; $n=5$ flare). All $P$ values in $\mathbf{C}$ are greater than 0.05 as calculated by unpaired $t$ test. See also Supplemental Figures 2 and 3.

drawal compared with those that flared (Figure 3A). Deconvolution of the HBV-specific $\mathrm{T}$ cell repertoire revealed that, while responses against $\mathrm{x}$ and envelope were present at low frequencies in both patient groups, those targeting core and polymerase could be detected at significantly higher frequencies (Figure $3 \mathrm{~B}$ ) and longitudinally at different time points (Figure 3, C and D, and Table 1) in patients for whom NUC therapy could be safely removed. Further analysis by intracellular cytokine staining demonstrated that HBV-specific $\mathrm{T}$ cells were mostly contained within the $\mathrm{CD} 8^{+} \mathrm{T}$ cell subset, with polymerase-specific $\mathrm{T}$ cells being present at higher frequencies compared with those specific for core (Figure 3, E and F, and Supplemental Figure 1B). In line with previous studies (18), the magnitude of the HBV-specific $\mathrm{T}$ cell response during hepatic flares appeared to be lower, or at least not superior, to that detected in patients that did not flare, suggesting that hepatic flares may not be driven by HBV-specific $\mathrm{T}$ cells. Thus, this comprehensive analysis of HBV-specific T cells shows that, during NUC therapy, the detection of core and polymerase-specific $\mathrm{T}$ cells that possess in vitro proliferative ability can predict whether patients may safely withdraw from NUC therapy (Figure 3, G and H).

Characterization of immune populations and transcriptional profiles associated with HBV control. We next investigated ex vivo whether non-HBV-specific components of the human immune system could be associated with HBV control after NUC therapy discontinuation. Recent studies have suggested that the frequency of NK cell populations may represent a potential marker for 
A Relative expression (Z score)

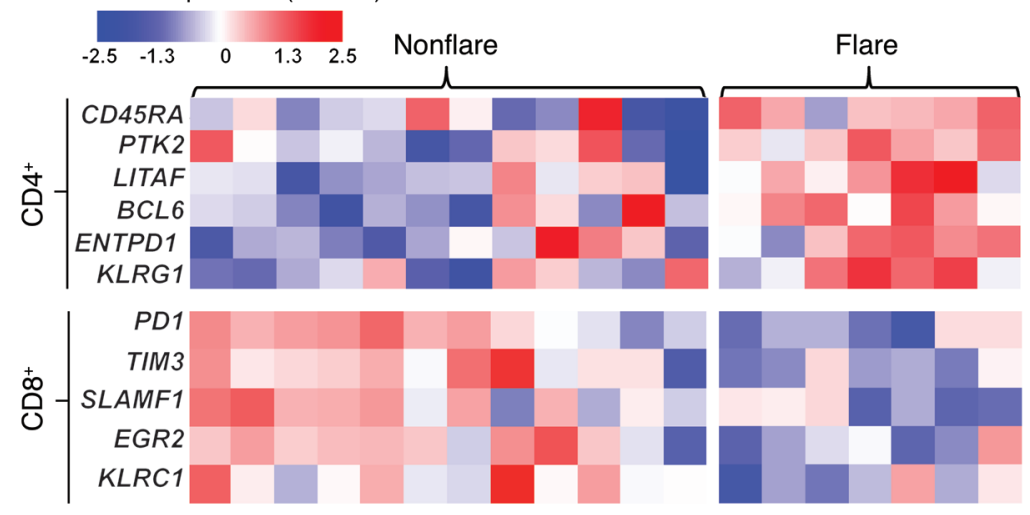

B

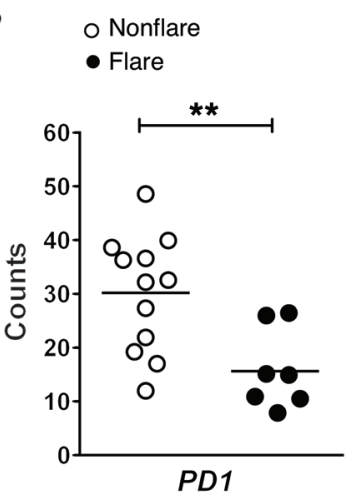

C
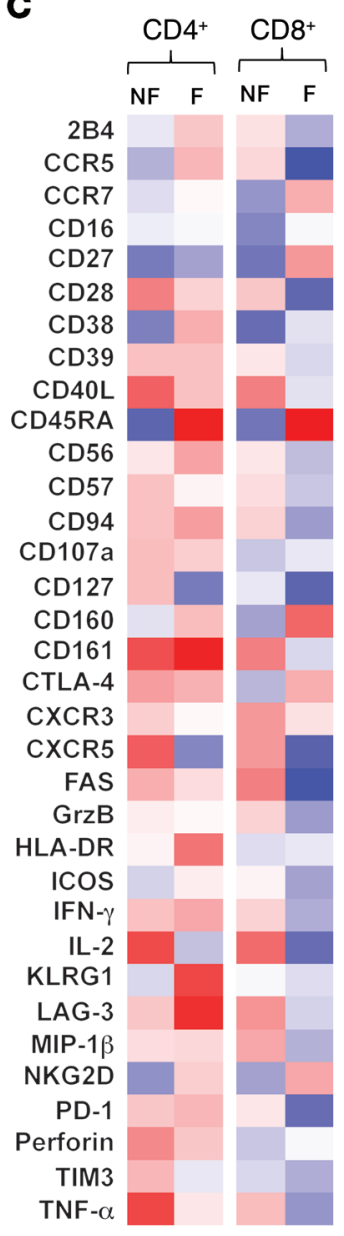

Relative expression ( $Z$ score)

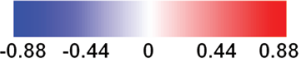

D

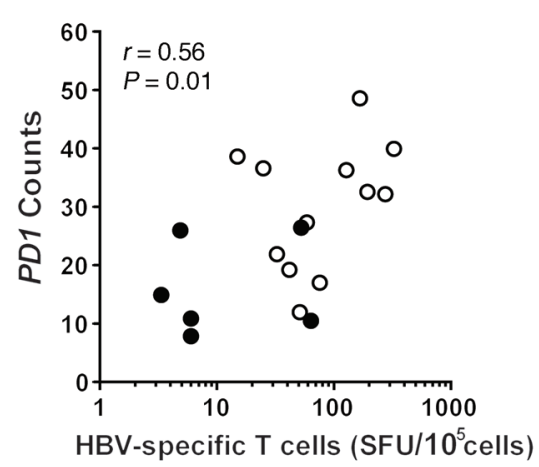

$\mathbf{F}$
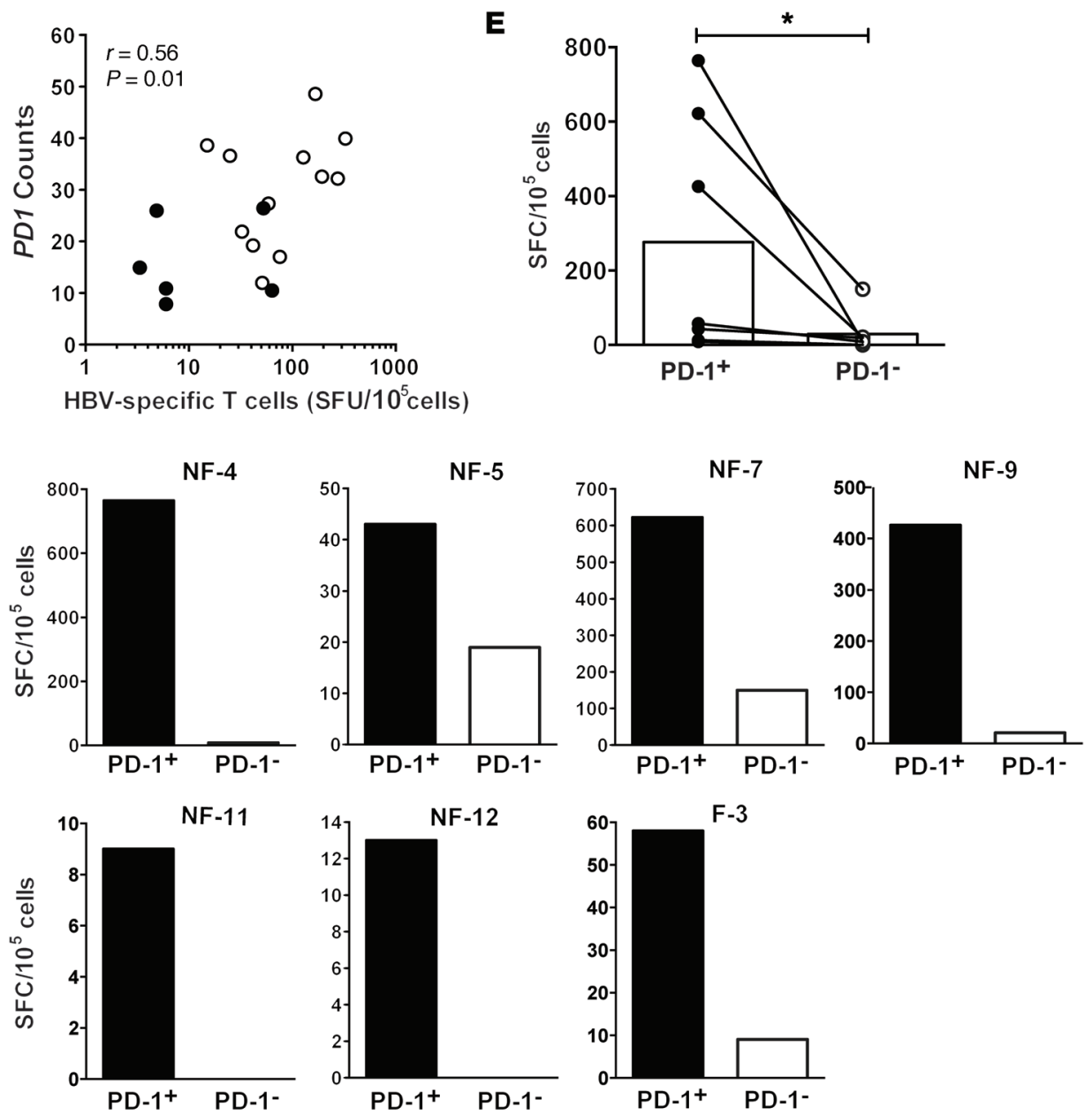

G

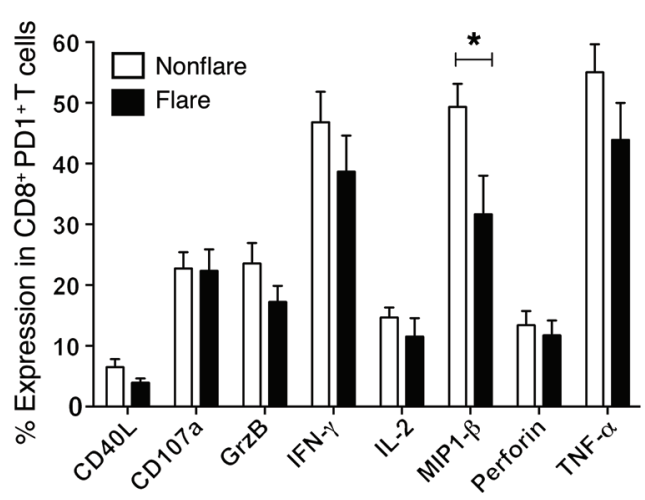


Figure 5. Functional HBV-specific $T$ cells are enriched in the $P D-1^{+} T$ cell fraction. (A-C) mRNA expression of immune-regulatory genes was analyzed by NanoString in $\mathrm{CD} 4^{+}$and $\mathrm{CD} 8^{+} \mathrm{T}$ cells sorted from the peripheral blood of patients that flared $(n=7)$ or did not flare $(n=12)$ upon therapy discontinuation. The differentially expressed genes ( $>1.5$-fold difference, $P<0.05)$ in $\mathrm{CD}^{+}$and $\mathrm{CD} 8^{+} \mathrm{T}$ cells from the 2 patient groups are shown by heatmap (A). $P D 1$ expression by $C D 8^{+} \mathrm{T}$ cells is also shown as normalized NanoString counts (B). Statistics are calculated by nonparametric, 2-tailed Mann-Whitney $U$ test. The expression of immune-regulatory markers by $\mathrm{CD}^{+}$and $\mathrm{CD} 8^{+} \mathrm{T}$ cells from patients that did not flare or from those that flared upon therapy withdrawal was evaluated by CyTOF (C). Relative expression of each marker is plotted as z-score calculated for each experiment ( $n=12$ nonflare; $n=5$ flare; see Methods). All differences are not statistically significant after correcting for multiple comparisons with HolmŠidák or Šidák-Bonferroni method. (D) Pearson correlation of PD1 mRNA expression in total $C D 8^{+} T$ cells and frequencies of HBV-specific T cells. (E-F) PD-1+ and PD-1- T cells were sorted ex vivo from peripheral blood of patients ( $n=7: n=6$ nonflare, $n=1$ flare) and expanded in the presence of autologous antigen-presenting cells pulsed with HBV peptides. Expanded PD-1+ and PD-1- cells were tested for recognition of HBV peptides by IFN- $\gamma$. Data are summarized for 7 patients $(\mathbf{E})$ and are shown for single patients (F). The ability of PD- $1^{+} \mathrm{CD} 8^{+} \mathrm{T}$ cells to produce cytokines and effector molecules following polyclonal stimulation was assessed by CYTOF in PBMCs of patients from the 2 groups ( $n=12$ nonflare; $n=5$ flare) (C). Statistics were calculated using the nonparametric, 2-tailed Mann-Whitney $U$ test except for in $\mathbf{E}$, where the nonparametric Wilcoxon matched-pairs signed rank test was used. ${ }^{*} P \leq 0.05 ;{ }^{* *} P \leq 0.01$.

NUC suspension (11) and the identification of an immunological signature directly ex vivo would provide a more efficient and easily reproducible approach. Two distinct strategies were adopted. First, we utilized CyTOF to uncover the phenotypic and functional properties of peripheral immune cells. Second, mRNA expression analysis was performed to determine the genes involved in immune pathways in purified $\mathrm{CD} 4^{+}$and $\mathrm{CD} 8^{+} \mathrm{T}$ cell subsets by NanoString technology.

The expression of 40 parameters involved in $\mathrm{T}, \mathrm{B}$, and NK cell activation, differentiation, and exhaustion (Supplemental Table 1) was evaluated by CyTOF. To uncover the functional capacity of these cells, PBMCs were analyzed after a brief polyclonal stimulation followed by intracellular cytokine staining. An unsupervised analysis was performed by $t$-distributed stochastic neighbor embedding ( $t$-SNE), an algorithm that maps cells with similar properties in close proximity, as previously described (19) (Figure 4A). To further visualize and characterize the populations of interest within the $t$-SNE maps, manual gating was performed (Figure 4, A and B; gating strategy shown in Supplemental Figure 2). The expression of immune markers was investigated in the distinct populations (Figure 4C). These analyses demonstrate that, during NUC therapy, both patient groups displayed T, B, and NK cell populations of similar frequencies and phenotypic/functional properties (Figure 4 and Supplemental Figure 3). The only exception was represented by $\mathrm{CD} 4^{+} \mathrm{T}$ cells, since their frequencies were increased in patients that did not flare compared with those that flared (Supplemental Figure 3). However, there was a substantial overlap of the frequencies of $\mathrm{CD} 4^{+} \mathrm{T}$ cells among the 2 groups, and only half ( 5 out of 10 ) of the patients who did not flare upon therapy discontinuation displayed $\mathrm{CD} 4^{+} \mathrm{T}$ cell frequencies that were higher compared with those observed in the patients that flared (Supplemental Figure 3).
We next investigated by NanoString the transcriptional profile of 579 selected immune genes in $\mathrm{CD}^{+}$and $\mathrm{CD} 8^{+} \mathrm{T}$ cells sorted ex vivo from the peripheral blood of patients during NUC viral suppression. Patients that did not flare upon treatment withdrawal displayed increased expression in $\mathrm{CD}^{+} \mathrm{T}$ cells of mRNAs encoding for the costimulatory proteins/inhibitory receptors (20) PD-1, TIM3, EGR2, KLRC1, and SLAMF1 (Figure 5, A and B). Furthermore, these patients exhibited decreased expression in $\mathrm{CD} 4^{+} \mathrm{T}$ cells of mRNAs encoding for immune markers, including CD45RA, BCL6, KLRG1, and CD39 (Figure 5A). A similar trend toward higher expression of PD-1 and TIM3 was observed by CyTOF in $\mathrm{CD}^{+} \mathrm{T}$ cells of patients that did not flare (Figure $5 \mathrm{C}$ ). Similarly, at the protein level, CD45RA and KLRG1 displayed a trend toward decreased expression in $\mathrm{CD} 4^{+} \mathrm{T}$ cells of patients that did not flare (Figure 5C). The other markers that were differentially expressed at the mRNA level in the 2 patient groups (Figure 5A) were not present in the CyTOF panel.

$H B V$-specific $T$ cells are enriched in the $P D-1^{+} T$ cell fraction. Among the differentially expressed genes, $P D 1$ was upregulated with the highest significance $(P=0.009)$ in $\mathrm{CD} 8^{+} \mathrm{T}$ cells of patients that did not flare, suggesting that an upregulation of PD-1 on $\mathrm{T}$ cells might be beneficial. Since recent studies have proposed an additional unappreciated role of PD-1 in the long-term survival of antigen-specific $\mathrm{CD} 8^{+} \mathrm{T}$ cells (21), we hypothesized that overexpression of PD-1 on T cells may allow HBV-specific T cells to escape deletion induced by chronic viral antigen stimulation. First, we noted that the levels of $P D 1$ in total $\mathrm{CD} 8^{+} \mathrm{T}$ cells correlated with the frequencies of in vitro-expanded HBV-specific $\mathrm{T}$ cells (Figure 5D). We then tested directly to determine whether $\mathrm{HBV}$-specific $\mathrm{T}$ cells were enriched in the $\mathrm{PD}-1^{+} \mathrm{T}$ cell fraction. PD- $1^{+}$and PD-1- $\mathrm{T}$ cells were sorted ex vivo from patient PBMCs, cocultured with core and polymerase peptide-pulsed autologous antigen-presenting cells for 10 days, and tested for the presence of HBV-specific T cells by IFN- $\gamma$ ELISPOT. Our results show that $\mathrm{HBV}$ core and polymerase-specific $\mathrm{T}$ cells were significantly enriched in the PD-1+ $1^{+}$fraction compared with the PD-1 counterpart in patients that did not flare upon therapy discontinuation $(n=6)$ and in a single patient from the flare group (only 2 patients from the flare group had detectable HBV-specific T cell responses, but PBMCs during NUC therapy were available for only 1 of these 2 patients). Data are summarized for 7 patients in Figure 5E and are shown for each patient in Figure 5F.

Finally, analysis by CyTOF of PBMCs following brief polyclonal stimulation showed a tendency toward higher cytokine-producing capabilities of $\mathrm{CD} 8^{+} \mathrm{PD}-1^{+} \mathrm{T}$ cells from patients that did not flare as compared with those that flared (Figure 5G). Taken together, these data suggest that PD-1 expression on T cells may help to preserve a population of partially exhausted $\mathrm{T}$ cells specific for selected HBV antigens.

Validation in an independent patient cohort of chronic $\mathrm{HBV}$ patients undergoing single NUC therapy. This comprehensive immunological analysis was performed on an initial cohort of 19 HBeAg-negative chronic HBV patients under long-term combination therapy (TDF/LAM) followed by 1 year of monotherapy (LAM; cohort 1). We next sought to validate our findings in a second cohort of $27 \mathrm{HBeAg}$-negative chronic HBV patients, treated with a single NUC agent (entecavir [ETV], TDF, or telbivudine 
Table 2. Details of the patients from the validation cohort (cohort 2)

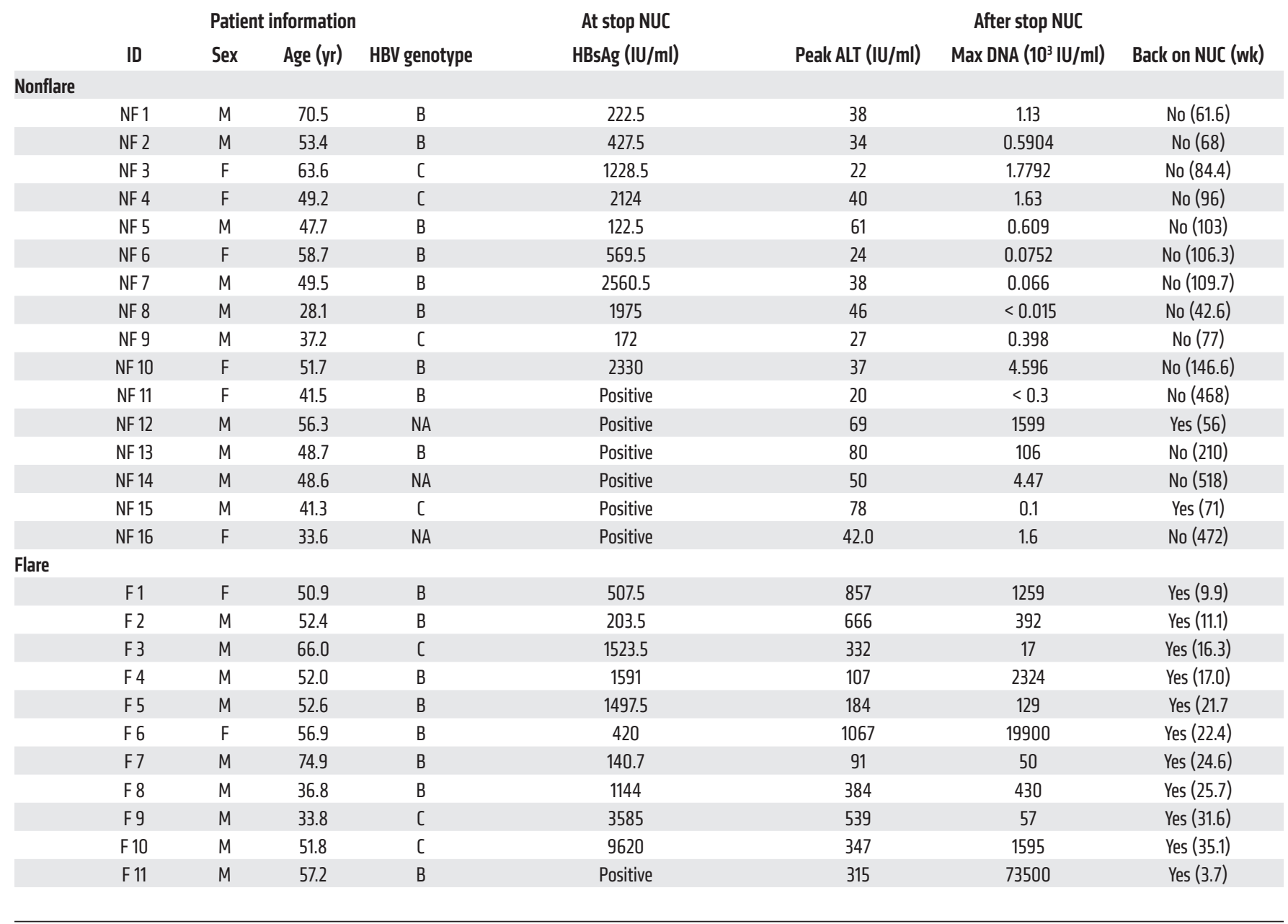

[LDT]; Figure 1, cohort 2). Of note, the 2 cohorts differed not only in the treatment regimes, but also in ethnicity and HBV-infecting genotype. Patients from cohort 1 were recruited in London (UK) and were heterogeneous in ethnicity and in HBV genotype of infection (A, B, C, D, or E). In contrast, patients from cohort 2 were recruited in Southeast Asia, were all of Han Chinese ethnicity, and were infected with $\mathrm{HBV}$ genotypes $\mathrm{B}$ or $\mathrm{C}$, the predominant $\mathrm{HBV}$ genotypes present in this region (22) (Tables 1 and 2).

The clinical and virological profiles of the chronic HBV patients of cohort 2 are shown in Table 2. An identical grouping of flare versus nonflare patients based on similar parameters set for cohort 1 was adopted. Nonflare and flare patients were defined based on serum ALT values below ( $\leq 80 \mathrm{IU} / \mathrm{ml}$ ) or above (>80 IU/ ml) $2 \times$ ULN, respectively, during an 8-month (35 week) period subsequent to treatment withdrawal.

Due to the limited number of cryopreserved PBMCs available for patients in cohort 2 while they were on NUC therapy, we focused our immunological analysis on the characterization of HBV-specific $T$ cells after in vitro expansion with overlapping HBV peptides and on the analysis of mRNA gene expression on purified $\mathrm{CD}^{+}$and $\mathrm{CD}^{+} \mathrm{T}$ cells. A higher frequency of HBV-specific T cells was detected in those patients, while undergoing NUC therapy, that did not flare upon therapy withdrawal, confirming the findings from cohort 1 in this second cohort (Figure 6A). Similarly to what was observed in cohort 1 , deconvolution of the HBV-specific $\mathrm{T}$ cell repertoire revealed that, while responses against $\mathrm{x}$ and envelope were present at low frequencies in both patient groups, those targeting core and polymerase could be detected at higher frequencies in patients that did not flare (although this only reached statistical significance for core; Figure 6B). We next asked whether HBV-specific T cells were contained preferentially within the $\mathrm{PD}-1^{+}$fraction, as was observed for cohort 1. PD $-1^{+}$and PD-1 ${ }^{-}$T cells were sorted directly ex vivo from PBMCs of 6 patients on NUC therapy that did not flare upon therapy withdrawal, and cells were subsequently expanded with peptides spanning HBV core and polymerase proteins. After 10 days, PD- $1^{+}$ and PD-1 ${ }^{-}$cells were tested for HBV recognition by IFN- $\gamma$ ELISPOT. Similarly in this cohort, HBV-specific T cells could be expanded preferentially from the $\mathrm{PD}-1^{+}$fraction, as we had demonstrated for patients in cohort 1 (data summarized for $n=6$ patients in Figure $6 \mathrm{C}$ and shown for the individual patients in Figure 6D).

However, mRNA gene expression by NanoString on purified $\mathrm{CD}^{+}$and $\mathrm{CD}^{+} \mathrm{T}$ cells did not confirm the differential expression of $P D 1$ in $C D 8^{+} \mathrm{T}$ cells in patients that did not flare compared with those that flared, and $P D 1$ expression levels were low in both 

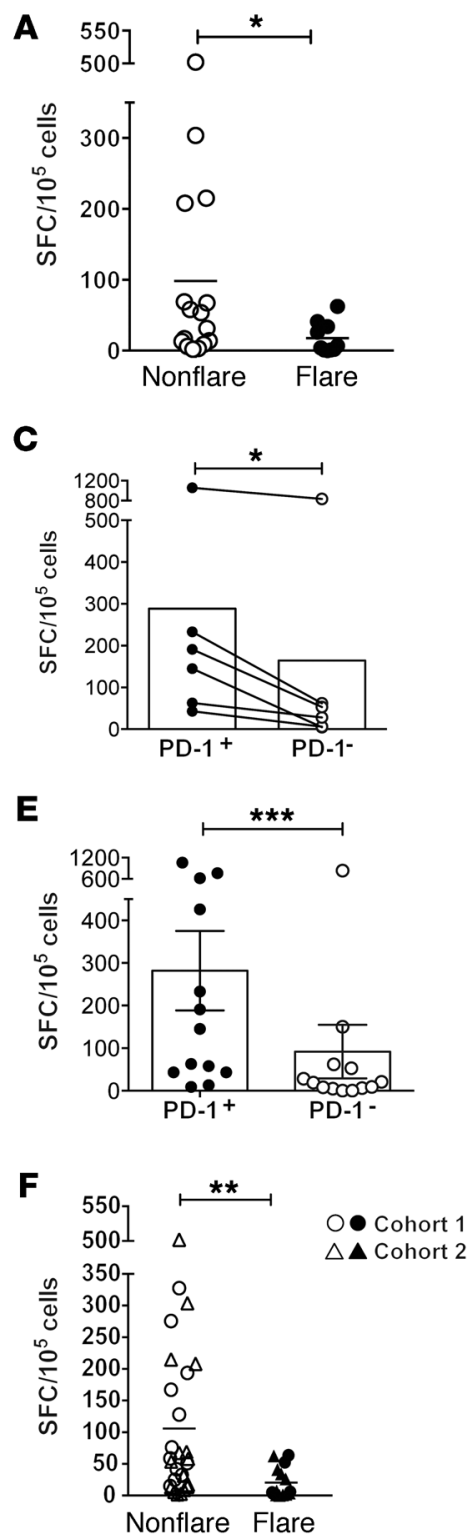
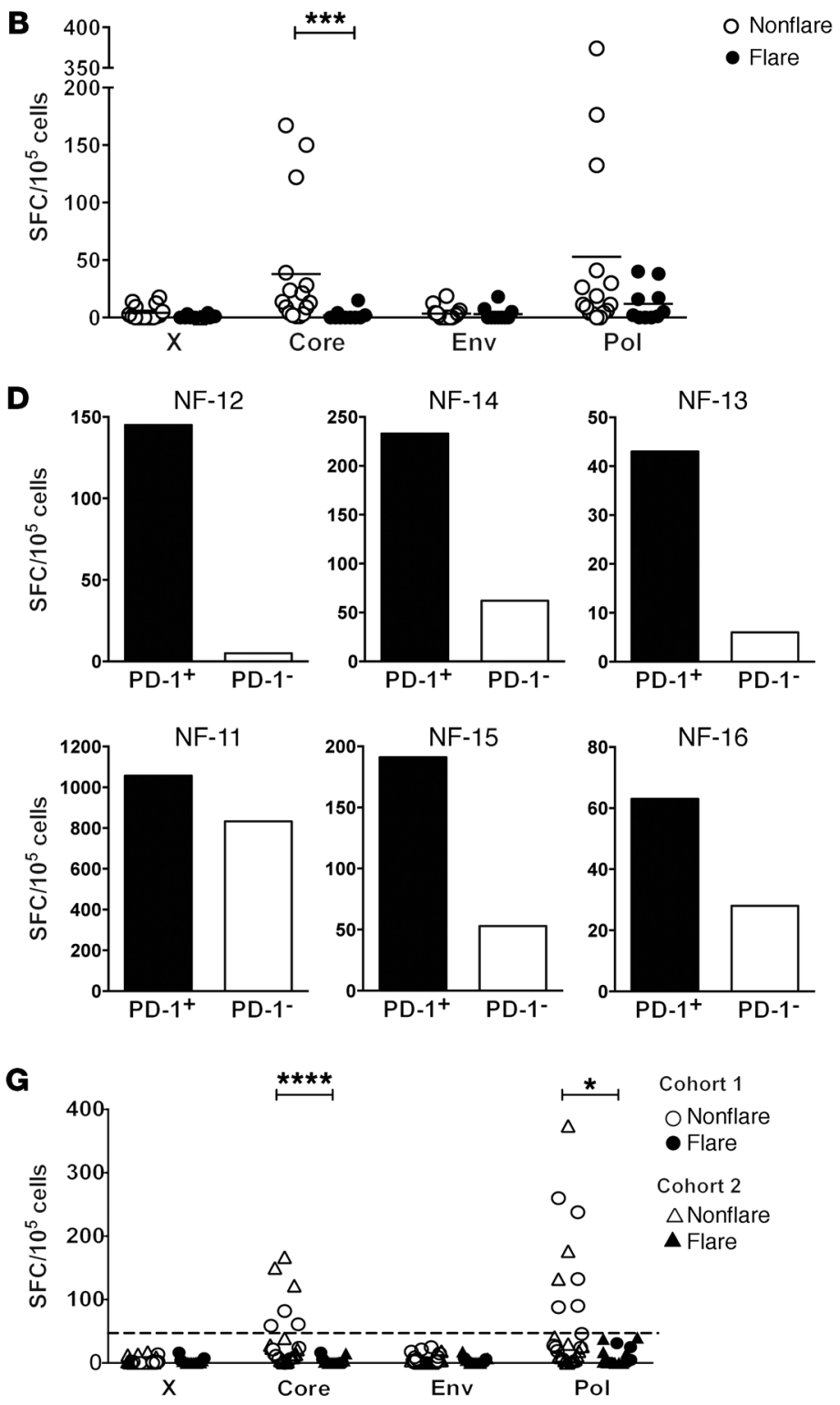

Figure 6. Validation in an Asian patient cohort (cohort 2) of the increased frequencies of circulating HBV core and polymerase-specific T cells in chronic HBV patients undergoing NUC therapy that did not flare upon therapy discontinuation. HBV-specific T cells were assessed after 10-day expansion of patient PBMCs with a panel of overlapping 15-mer peptides spanning the HBV proteome, followed by IFN- $\gamma$ ELISPOT in the presence of peptides pooled according to the single proteins ( $x$, core, env, and pol). Results are expressed as spot-forming cells relative to $10^{5}$ PBMCs. (A) Total HBV-specific T cell responses during NUC therapy $(n=26)$. (B) Deconvolution of the HBV-specific T cell response from $\mathbf{A}$ into single HBV proteins. (C) PD- $1^{+}$and PD-1- T cells were sorted ex vivo from peripheral blood of patients $(n=6)$ and expanded in the presence of autologous antigen-presenting cells pulsed with HBV core and polymerase peptides. Expanded PD-1+ and PD-1- cells were tested for recognition of HBV peptides by IFN- $\gamma$. Data are summarized for 6 patients (C) and are shown for the single patients (D). (E-C) Pooled data from cohorts 1 and 2 show IFN- $\gamma$ production of ex vivo-sorted PD-1+ and PD-1- cells after in vitro expansion ( $n=13 ; \mathbf{E}$ ), total HBV-specific T cell responses during therapy $(n=43 ; \mathbf{F})$, and the HBV-specific T cell response to single HBV proteins (C). The dotted line in $\mathbf{G}$ marks the threshold of HBV-specific T cell response associated with absence of hepatic flares upon therapy withdrawal. Circle and triangle symbols in $\mathbf{F}$ and $\mathbf{G}$ represent patients from cohort 1 and 2, respectively. Statistics were calculated using the nonparametric, 2-tailed Mann-Whitney $U$ test except for in $\mathbf{C}$ and $\mathbf{E}$, where the nonparametric Wilcoxon matched-pairs signed rank test was used. ${ }^{*} P \leq 0.05 ;{ }^{* *} P \leq 0.01 ;{ }^{* *} P \leq 0.001$; ${ }^{* * * *} P \leq 0.0001$.

patient groups. Of the 579 immune genes that were analyzed by NanoString, 122 genes were differently expressed in $\mathrm{CD} 4^{+} \mathrm{T}$ cells and 137 in $\mathrm{CD}^{+} \mathrm{T}$ cells between the 2 groups of patients tested while undergoing NUC therapy (Supplemental Figure 4). Furthermore, the frequencies of $\mathrm{CD}^{+}$and $\mathrm{CD}^{+} \mathrm{T}$ cells were not different in patients that did and did not flare upon therapy discontinuation (Supplemental Figure 5).
Pooled data from cohorts 1 and 2 showing the enrichment of HBV-specific T cells in the PD- $1^{+} \mathrm{T}$ cell fraction is shown in Figure $6 \mathrm{E}$. Pooled data from the 2 cohorts showing the increased frequencies of HBV-specific T cells, in particular of those targeting core and polymerase, in patients that did not flare following therapy withdrawal is shown in Figure 6, F and G. Of note, our data identify a threshold of HBV core or polymerase-specific T cell frequen- 
cies ( $>40$ SPC $/ 10^{5}$ cells) that is associated with HBV control in the absence of severe hepatic flare upon therapy discontinuation (Figure 6G, dotted line). Collectively, our results across 2 distinct patient cohorts suggest that if patients display a HBV core and/ or polymerase-specific $\mathrm{T}$ cell response above $40 \mathrm{SPC} / 10^{5}$ cells, they have a high likelihood of controlling $\mathrm{HBV}$ without developing severe hepatic flares.

In conclusion, our study demonstrates, in 2 independent cohorts of chronic HBV patients undergoing long-term NUC therapy, that increased frequencies of functional PD $-1^{+}$HBV polymerase/core-specific $\mathrm{T}$ cells correlate with the absence of hepatic flares upon NUC therapy withdrawal.

\section{Discussion}

Advanced antiviral agents for the treatment of chronic HBV infection are in the development pipeline. However, the implementation of these new treatments is often complicated by the current guidelines, which have recommended maintaining NUC therapy indefinitely. Discontinuation of NUCs, the current treatment of choice for chronic HBV patients, is associated with high rates of virological relapse and severe hepatic inflammation (23). Previous studies have suggested that NUC therapy can be safely removed only in the very few patients who have experienced HBsAg seroconversion or displayed values of HBsAg levels below $100 \mathrm{IU} / \mathrm{ml}$ (7). Thus, the identification of biological markers that predict whether NUC therapy can be safely interrupted is urgently needed to move forward in the clinical management of chronic HBV patients.

To achieve this goal, we characterized antigen-specific and global immunological parameters in 2 independent cohorts of chronic HBV patients undergoing different regimes of NUC therapy. We show that patients who control HBV after therapy withdrawal without triggering severe hepatic inflammation are characterized, during the course of NUC therapy, by increased frequencies of HBV core and polymerase-specific T cells. This represents, to our knowledge, the first study to report a correlation of the quantity and function of $\mathrm{HBV}$-specific $\mathrm{T}$ cells with the ability to control HBV replication after NUC therapy withdrawal. Our data are consistent with previous reports showing that the magnitude of the HBV-specific T cell response correlates with HBV control in adult chronic HBV patients (15).

In particular, our data demonstrate that HBV-specific T cells present in the blood of chronic HBV patients who do not flare are selectively enriched within the $\mathrm{PD}-1^{+} \mathrm{T}$ cell population and that these cells are functional, at least in terms of their proliferative capacity and ability to produce IFN- $\gamma$. Since PD- 1 expression is considered the classical hallmark of exhausted T cells (24), our results may appear counterintuitive. However, studies performed in lymphocytic choriomeningitis virus-infected (LCMV-infected) mice demonstrate that PD-1 expression on $\mathrm{T}$ cells allows the improved survival of $\mathrm{T}$ cell memory populations that would otherwise be deleted by the high antigenic load present during chronic viral infection (21). Similarly, recent studies show that patients with partial immune control of HBV infection display higher levels of intrahepatic PD $-1^{+} \mathrm{CD} 39^{+}$tissue-resident $\mathrm{CD} 8^{+} \mathrm{T}$ cells that possess the capacity to mount immediate and strong cytokine responses (25). PD-1 expression on $\mathrm{HBV}$-specific $\mathrm{T}$ cells might therefore favor their survival by limiting the excessive activation of these cells. Earlier studies showed that HBV-specific T cells could be detected in chronic HBV patients with lower levels of viremia and that these cells were mostly PD-1+(26).

Of note, in both patient cohorts, HBV-specific T cells target exclusively core and polymerase, but not the envelope protein. This finding may suggest that $\mathrm{HBV}$ envelope-specific $\mathrm{T}$ cells have been deleted during the course of chronic infection due to high antigenic loads. In contrast, the presence of PD $-1^{+} \mathrm{HBV}$ core and polymerase-specific $\mathrm{T}$ cells selectively in patients with $\mathrm{HBV}$ control suggests that these cells may retain their antiviral capacity and contribute to protection in vivo. However, only the direct analysis of these cells within the intrahepatic environment will provide direct evidence of their protective capacity and further our understanding of the mechanisms underlying HBV control in patients who do not relapse upon therapy withdrawal. It is evident that in these patients, HBV-specific T cells lack the ability to ultimately clear $\mathrm{HBV}$ infection and that, even after in vitro expansion, their magnitude is lower than that of HBV-specific T cells present in patients with resolved acute $\mathrm{HBV}$ infection. Previous studies have shown that the function of $\mathrm{PD}-1^{+} \mathrm{HBV}-$ specific $\mathrm{T}$ cells that are detectable in chronic HBV patients with lower levels of viremia could be improved in vitro by blocking PD-1/PD-L1 engagement (26). The implications of PD-1/PD-L1 therapy in vivo are, however, difficult to predict. Based on our findings, we speculate that a subgroup of HBV patients with detectable PD- $1^{+}$ HBV-specific $\mathrm{T}$ cells (such as those that did not flare following therapy discontinuation) may benefit from PD1/PD-L1-blockage therapy, as this may lead to full functional recovery of partially exhausted HBV-specific T cells. Further studies are, however, needed to understand these processes.

The primary goal of this work was the definition of an immunological marker for the safe discontinuation of NUC therapy. The association of $\mathrm{PD}-\mathrm{1}^{+} \mathrm{HBV}-$ specific $\mathrm{T}$ cells with $\mathrm{HBV}$ control in the absence of a hepatic flare is an important initial step forward toward achieving this goal. However, the methodology used for the analysis of antigen-specific $\mathrm{T}$ cells in vitro is lengthy, difficult to standardize, and requires specialized technical skills that are not available in every laboratory. Thus, we sought to identify a biomarker directly ex vivo by utilizing high-throughput immunological methods such as CyTOF and NanoString. Unfortunately, these efforts did not lead to the identification of a biomarker that could be validated across 2 independent cohorts. CyTOF analysis of PBMCs from patients of the first cohort revealed a correlation between increased frequencies of $\mathrm{CD}^{+} \mathrm{T}$ cells and HBV control, but this was not confirmed in the second cohort. More strikingly, the association of higher PD1 mRNA expression in total $\mathrm{CD}^{+} \mathrm{T}$ cells of patients from the first cohort who did not flare was also not confirmed in patients from the second cohort. The reasons underlying these discrepancies are not fully understood, but may be related to the different treatment regimes of the 2 cohorts. Patients from cohort 2 were treated with NUC analogues (e.g., LDT, TDF, and ETV) with higher antiviral potency than those used for the treatment of patients from cohort 1 (LAM). Since PD-1 expression on T cells, both at the protein and mRNA levels, is suppressed by NUC treatment in proportion to the level of HBV reduction (27), treatment with TDF and ETV may fully suppress $\mathrm{T}$ cell expression of PD-1 and thus completely mask the 
differences detected in cohort 1 . These considerations are in line with the low levels of PD1 mRNA expression detected in $\mathrm{CD}^{+} \mathrm{T}$ cells from patients of cohort 2. Of note, the work of McKinney et al. (12) shows that $\mathrm{T}$ cell exhaustion signatures predictive of disease outcomes were evident only during the active phases of diseases. Similarly, it is possible that the expression of inhibitory markers such as PD1 or TIM3 should be analyzed during the inflammatory/ active phase of disease, when $\mathrm{T}$ cells are undergoing activation, rather than during NUC therapy, when viral replication is inhibited. Unfortunately, the limited availability of PBMC samples from the 2 patient cohorts did not allow a longitudinal analysis of PD1 expression at sequential time points of the study.

In summary, the in-depth analysis using traditional and recently developed immunological assays identifies in 2 independent cohorts the presence of partially exhausted HBV core and polymerase-specific T cells as valuable biomarkers of HBV control after NUC therapy withdrawal. The implementation of a methodology that allows the detection of HBV-specific T cells directly ex vivo, without the prior need to expand the cells in vitro, might represent the necessary step to translate our findings into the clinic by developing a reliable, easy to perform, and rapid assay to safely identify patients for NUC withdrawal. The sensitivity of such an assay may be superior if performed on freshly isolated peripheral blood, as recent data suggests that the freezing/thawing process introduces functional modifications in $\mathrm{T}$ cells that negatively affect their functionality as assessed by IFN- $\gamma$ ELISPOT (Carlo Ferrari, personal communication). If successful, an ex vivo $\mathrm{T}$ cell assay could be easily translated into a clinical diagnostic test. For example, QuantiFERON-CMV is a whole blood assay based on the measurement of IFN- $\gamma$ production by T cells following recognition of human cytomegalovirus (HCMV) peptides. This kit is routinely used in the clinic to monitor T cell-mediated immunity to HCMV, which is critical for controlling reactivation of HCMV in the context of organ transplantation.

In conclusion, our study highlights the beneficial role of the expression of exhaustion markers on $\mathrm{T}$ cells during chronic viral infection, since PD-1 expression associates with the long-term persistence of virus-specific $\mathrm{T}$ cells in human chronic viral infection. Furthermore, our data propose that the levels of residual HBV-specific T cells present in patients during NUC therapy can be used to predict the safe discontinuation of NUC antiviral therapy.

\section{Methods}

Patient cohort 1 . Twenty-nine HBeAg-negative chronic HBV patients on long-term dual antiviral therapy including TDF and LAM were enrolled for this study. Patients were treated with dual NUC therapy for a minimum of 24 months (median treatment times 36 months; range 24-72 months), with patients virally suppressed for at least 18 months. TDF was then stopped and patients were maintained on LAM therapy only for an additional 12 months, after which all NUC therapy was discontinued in 21 patients. Patients were monitored routinely at 4-week intervals during and for up to 2 years after stopping therapy. Following NUC discontinuation, patients differentiated into 2 groups based on the absence or presence of hepatic flares during the 6 months ( 24 weeks) that immediately followed treatment withdrawal, as described in the main text. Enrollment criteria and patient details are shown in Figure 1 and Table 1.
Patient cohort 2. Twenty-seven HBeAg-negative chronic HBV patients on long-term antiviral therapy were enrolled for this study. Antiviral therapy consisting of ETV, TDF, or LDT was withdrawn, and patients were monitored routinely. Following therapy withdrawal, patients differentiated into 2 groups based on the presence or absence of hepatic flares during the 8 months ( 35 weeks) that immediately followed treatment withdrawal as described in the main text. Enrollment criteria and patient details are shown in Figure 1 and Table 2.

$H B V$ peptide library. Peptide libraries of 313 fifteen-mer peptides overlapping by 10 amino acids were used to identify HBV-specific $\mathrm{T}$ cells. The peptides covered the entire sequence of HBV genotypes B, C, and D (GenBank AF121243, AF 112063, AF 21241, respectively) and were purchased from Mimotopes. The purity of the peptides was above $80 \%$, and their composition was confirmed by mass spectrometry analysis. Peptides were pooled as described previously (13). The peptide libraries were matched to the closest HBV genotype of each patient, as indicated in Tables 1 and 2.

PBMC isolation and T cell culture. PBMCs were isolated from peripheral blood by Ficoll gradient and cryopreserved. Cells were thawed on the day of the experiment and were used directly for the ex vivo experiments (NanoString and CyTOF). For the in vitro assays, T cell lines were generated as follows: $20 \%$ of PBMCs were pulsed with $10 \mu \mathrm{g} / \mathrm{ml}$ of the overlapping $\mathrm{HBV}$ peptides for 1 hour at $37^{\circ} \mathrm{C}$, subsequently washed, and cocultured with the remaining cells in AIM-V medium (Gibco; Thermo Fisher Scientific) supplemented with $2 \%$ AB human serum (Gibco; Thermo Fisher Scientific). T cell lines were cultured for 10 days in the presence of $20 \mathrm{U} / \mathrm{ml}$ of recombinant IL-2 (R\&D Systems).

ELISPOT. ELISPOT assays for the detection of IFN- $\gamma$-producing cells were performed on in vitro-expanded T cell lines using HBV peptides pooled into the following mixtures: $\mathrm{X}$, core, envelope 1 (env 1), env 2, polymerase 1 (pol 1), pol 2, pol 3, pol 4. T cell lines were incubated overnight at $37^{\circ} \mathrm{C}$ with pools of $\mathrm{HBV}$ peptides $(5 \mu \mathrm{g} / \mathrm{ml})$, where final DMSO concentrations did not exceed $0.5 \%$. IFN- $\gamma$ ELISPOT assays (Millipore) were performed as described previously (28).

Intracellular staining. Expanded $\mathrm{T}$ cell lines were stimulated overnight at $37^{\circ} \mathrm{C}$ with or without HBV peptide pools $(5 \mu \mathrm{g} / \mathrm{ml})$ in the presence of $2 \mu \mathrm{g} / \mathrm{ml}$ brefeldin A (Sigma-Aldrich). As a positive control, cells were stimulated with PMA $(2 \mathrm{ng} / \mathrm{ml})$ and ionomycin $(1 \mu \mathrm{g} / \mathrm{ml})$. Cells were stained with the yellow LIVE/DEAD fixable dead cell stain kit (Invitrogen) and anti-CD3 V500 (clone UCHT1), anti-CD4 BV605 (clone SK3), and anti-CD8 APC CY7 (clone SK1) antibodies. Cells were subsequently fixed and permeabilized using the Cytofix/Cytoperm kit (BD Biosciences - Pharmingen) and stained with anti-IFN- $\gamma$ PE (clone 25723, R\&D Systems), anti-TNF- $\alpha$ AF 700 (clone MAb11), and anti-IL-2 PerCPCY5.5 (clone MQ1-17H12) antibodies and analyzed on a BD-LSR II FACS Scan. Data were analyzed by FlowJo (Tree Star Inc.). Antibodies were purchased from BD Biosciences - Pharmingen unless otherwise stated.

Cell sorting. PBMCs were thawed and used directly for cell sorting on a BD FACSAria, as follows. For the NanoString experiments, PBMCs were stained with the Near-IR LIVE/DEAD fixable dead cell stain kit (Invitrogen), anti-CD3 V500 (clone UCHT1), anti-CD4-PE (clone SK3), and anti-CD8-PECY7 (clone RPA-T8) antibodies. CD4 ${ }^{+}$ and $\mathrm{CD} 8^{+} \mathrm{T}$ cells were purified based on expression of $\mathrm{CD} 3, \mathrm{CD} 4$, and CD8 to a purity of greater than $95 \%$. For the PD-1 experiments, cells were stained with the Near-IR LIVE/DEAD fixable dead cell stain kit (Invitrogen) and anti-CD3 FITC (clone APA 1/1) and anti-CD279 (PD- 
1) BV 421 (clone EH12.2H7) antibodies. Live CD3 ${ }^{+} \mathrm{PD}^{-1^{+}}, \mathrm{CD}^{+} \mathrm{PD}-1^{-}$, and $\mathrm{CD}^{-}$populations were purified by cell sorting according to $\mathrm{CD} 3$ and PD-1 expression to a purity of greater than $95 \%$. Sorted $\mathrm{CD}^{-}$cells were pulsed with $\mathrm{HBV}$ polymerase peptides from the corresponding HBV genotype for 1 hour at $37^{\circ} \mathrm{C}$, washed, and mixed 1:1 with unpulsed $\mathrm{CD}^{-}$cells. $\mathrm{CD}^{-}$cells were cocultured for 10 days with autologous PD $-1^{+}$and PD-1- $\mathrm{T}$ cells at a 1:1 ratio in the presence of IL-2 $(20 \mathrm{U} / \mathrm{ml})$. The presence of HBV-specific T cells in PD-1 ${ }^{+}$and $\mathrm{PD}-1^{-}$fractions was assessed by IFN- $\gamma$ ELISPOT. Antibodies were purchased from BD Biosciences - Pharmingen unless otherwise stated.

NanoString gene expression analysis. $\mathrm{CD}^{+}$and $\mathrm{CD} 8^{+} \mathrm{T}$ cell subsets purified by cell sorting were lysed in RLT lysis buffer (QIAGEN, supplemented with 2-mercaptoethanol at 1:100) at a ratio of $1 \mu \mathrm{l}$ RTL:10,000 cells. Cell lysates from a minimum of 20,000 cells were analyzed using the preassembled nCounter GX Human Immunology Kit and the nCounter system (NanoString Technologies) according to the manufacturer's instructions. Samples were then normalized based on the geometric means of both the supplied positive controls and the panel of housekeeping genes, as recommended by the manufacturer. Only genes that were significantly different $(P<0.05, t$ test, false discover rate [FDR] adjusted) and at least 1.5-fold differentially expressed between the 2 groups of patients were considered. For heatmap representation, the expression level of each gene was $\log _{2}$, which was then $\mathrm{z}$-score transformed using a custom R script.

CyTOF. PBMCs were thawed, washed with prewarmed complete RPMI, and rested overnight at $37^{\circ} \mathrm{C}$. The following day, cells were washed and stained at $37^{\circ} \mathrm{C}$ in 96 -well tissue culture-treated round-bottom plates (BD Falcon) with the anti-CD107a antibodies (Supplemental Table 1) for 30 minutes prior to stimulation. Cells were then stimulated for 4 hours with PMA (150 ng/ml) and ionomycin (1 $\mu \mathrm{M})$ in the presence of brefeldin A and monensin (eBioscience). After stimulation, cells were washed twice in cold CyFACS buffer (PBS $4 \%$ FBS, 2 mM EDTA, 0.05\% sodium azide) and incubated for 5 minutes on ice with Cisplatin (Pt-195, $200 \mu \mathrm{M}$, MilliporeSigma) for the measurement of cell viability. Cells were washed and stained with the surface antibody cocktail (Supplemental Table 1) on ice for 30 minutes. Cells were washed twice with CyFACS, once with PBS, and then fixed in PBS with 2\% paraformaldehyde (Electron Microscopy Sciences) at $4^{\circ} \mathrm{C}$. The next day, cells were washed with CyFACS, then incubated for 10 minutes with permeabilization buffer (BioLegend) and stained with an intracellular antibody cocktail in permeabilization buffer (Supplemental Table 1). After 45 minutes at room temperature, cells were washed once with permeabilization buffer and once with PBS and then incubated with dual mass-tag cellular barcodes, as previously described (29). After 30 minutes on ice, cells were washed once with permeabilization buffer and twice with CyFACS. Cellular DNA was labeled at room temperature with iridium interchelator $(250 \mathrm{nM}$, Fluidigm) diluted in PBS with 2\% paraformaldehyde for 20 minutes. Subsequently, cells were washed twice with CyFACS and twice in MilliQ water. Mass cytometry acquisition was performed on a CyTOF2 instrument. All purified antibodies that lacked carrier proteins were purchased from the companies listed in Supplemental Table 1. Antibody conjugation was performed according to the protocol provided by Fluidigm (see Supplemental Figure 2 for each staining).

CyTOF data analysis. After mass cytometry acquisition, the signal of each parameter was normalized based on the EQ beads (Fluidigm) as previously described (30). The 0 values were then random- ized using a uniform distribution of values between -1 and 0 in an $\mathrm{R}$ script. Cells were manually debarcoded using FlowJo. Live lymphocytes from different donors were randomly downsampled to 50,000 events for subsequent high-dimensional data analysis to normalize the contribution between donors. Samples were then used for $t$-SNE analysis similar to that previously described $(19,29)$ using custom $R$ scripts based on the "flowCore" and "Rtsne" (using CRAN R packages that perform the Barnes-Hut implementation of $t$-SNE) (31). In $\mathrm{R}$, all data were transformed using the "logicleTransform" function (flowCore package) with the parameters $w=0.25, t=16409, m=4.5$, and $a=0$ to roughly match scaling historically used in FlowJo. The pie charts (showing average relative frequencies) were generated using GraphPad Prism. Heat plots were generated using a custom R script after determining the frequency of positive expression using gating in FlowJo. For comparison of mean expression of markers on total $\mathrm{CD}^{+}$and $\mathrm{CD} 8^{+} \mathrm{T}$ cells, to control for variation in antibody and instrument sensitivity between experiments (5-9 patients plus 1 or 2 healthy donors tested per experiment), we calculated z-scores for each phenotypic marker for each batch by subtracting the average and dividing by the SD.

Statistics. Statistical significance was determined by GraphPad Prism using the nonparametric, 2-tailed Mann-Whitney $U$ test or as otherwise stated in the figure legend. Differences were considered significant at $P \leq 0.05$.

Study approval. All clinical investigations were conducted according to Declaration of Helsinki principles. For cohort 1, clinical assessment and peripheral blood sampling were performed during hepatitis treatment at the Royal London Hospital (London, UK). Written, informed consent was obtained prior to treatment discontinuation, and the study was approved by the Barts and the London NHS Trust local ethics review board and the NRES Committee London-Research Ethics Committee (reference 10/H0715/39). For cohort 2, clinical assessment and peripheral blood sampling were performed during hepatitis treatment at the National Taiwan University Hospital $(n=20)$ or the National University Hospital of Singapore (Singapore) $(n=7)$. Written, informed consent was obtained prior to treatment discontinuation, and the study was approved by the Institutional Review Board of National Taiwan University Hospital (201209022RIC) and by the Singapore Ethics Committee (2008/005700).

\section{Author contributions}

AB, PTFK, GRF, and USG designed the study. AB, PTFK, and EWN provided funding. LR and NLB designed the experiments. EWN and $\mathrm{YC}$ designed and provided expertise for CyTOF experiments. LR, NLB, USG, KK, and DZMT performed the experiments. LR, NLB, and EB analyzed the data. KK and DZMT provided technical support. EB performed statistical analysis. PTFK, USG, NKH, JHK, TCT, THS, and SGL recruited patients, performed clinical monitoring, and provided clinical expertise. LR, USG, and AB wrote the manuscript, and all authors provided critical review and approved the manuscript.

\section{Acknowledgments}

We would like to thank the Hepatology Services at The Royal London Hospital, Barts Health NHS Trust, including Louise Payaniandy, Deva Payaniandy, and Valerie Ross, who have supported and contributed to this work. We thank Wang Chi-Chang at National 
Taiwan University Hospital for laboratory assistance, and we thank and are grateful to Adeline Chia for her invaluable technical help. We appreciate and would like to thank all patients for participating in this study. This work was supported by a Singapore Translational Research (STaR) investigator award (NMRC/STaR/013/2012 to $\mathrm{AB}$ ) and by the Eradication of HBV TCR Program (NMRC/ TCR/014-NUHS/2015 to AB and EWN), Singapore Immunology Network (SIgN) core funding (to EWN), A-STAR/SIgN immuno- monitoring platform funding (to EWN), a Wellcome Trust Clinical Research Training Fellowship (107389/Z/15/Z to USG) and a Barts and The London Charity Large Project grant (723/1795 to PTFK).

Address correspondence to: Antonio Bertoletti, Emerging Infectious Diseases Program, DUKE-NUS Medical School, 8 College Road, Singapore 169857. Phone: 65.6601.3574; Email: antonio@ duke-nus.edu.sg.
1. Stanaway JD, et al. The global burden of viral hepatitis from 1990 to 2013: findings from the Global Burden of Disease Study 2013. Lancet. 2016;388(10049):1081-1088.

2. Seeger C, Mason WS. Hepatitis B virus biology. Microbiol Mol Biol Rev. 2000;64(1):51-68.

3. Höner Zu Siederdissen C, et al. Viral and host responses after stopping long-term nucleos ( $\mathrm{t}$ ) ide analogue therapy in HBeAg-negative chronic hepatitis B. J Infect Dis. 2016;214(10):1492-1497.

4. Gill US, et al. Assessment of bone mineral density in tenofovir-treated patients with chronic hepatitis B: can the fracture risk assessment tool identify those at greatest risk? J Infect Dis. 2015; 211(3):374-382.

5 . Sheldon J, et al. Selection of hepatitis B virus (HBV) vaccine escape mutants in HBV-infected and HBV/HIV-coinfected patients failing antiretroviral drugs with anti-HBV activity. J Acquir Immune Defic Syndr. 2007;46(3):279-282.

6. European Association for the Study of the Liver. Electronic address: easloffice@easloffice.eu, European Association for the Study of the Liver. EASL 2017 Clinical Practice Guidelines on the management of hepatitis B virus infection. J Hepatol. 2017;67(2):370-398.

7. Hadziyannis SJ, Sevastianos V, Rapti I, Vassilopoulos D, Hadziyannis E. Sustained responses and loss of HBsAg in HBeAg-negative patients with chronic hepatitis B who stop long-term treatment with adefovir. Gastroenterology. 2012;143(3):629-636.e1.

8. Berg T, et al. Long-term response after stopping tenofovir disoproxil fumarate in non-cirrhotic HBeAg-negative patients - FINITE study. J Hepatol. 2017;67(5):918-924.

9. Bertoletti A, Ferrari C. Adaptive immunity in HBV infection. J Hepatol. 2016;64(1 Suppl):S71-S83.

10. Maini MK, Peppa D. NK cells: a double-edged sword in chronic hepatitis B virus infection. Front
Immunol. 2013;4:57.

11. Boni C, et al. Natural killer cell phenotype modulation and natural killer/T-cell interplay in nucleos ( $\mathrm{t}$ )ide analogue-treated hepatitis e antigen-negative patients with chronic hepatitis $\mathrm{B}$. Hepatology. 2015;62(6):1697-1709.

12. McKinney EF, Lee JC, Jayne DR, Lyons PA, Smith KG. T-cell exhaustion, co-stimulation and clinical outcome in autoimmunity and infection. Nature. 2015;523(7562):612-616.

13. Tan AT, et al. Host ethnicity and virus genotype shape the hepatitis B virus-specific T-cell repertoire. J Virol. 2008;82(22):10986-10997.

14. Newell EW, Davis MM. Beyond model antigens: high-dimensional methods for the analysis of antigen-specific T cells. Nat Biotechnol. 2014;32(2):149-157.

15. Webster GJ, et al. Longitudinal analysis of CD ${ }^{+}$ $T$ cells specific for structural and nonstructural hepatitis B virus proteins in patients with chronic hepatitis B: implications for immunotherapy. J Virol. 2004;78(11):5707-5719.

16. Thimme R, et al. CD8 (+) T cells mediate viral clearance and disease pathogenesis during acute hepatitis B virus infection. J Virol. 2003;77(1):68-76.

17. Boni C, et al. Transient restoration of anti-viral $\mathrm{T}$ cell responses induced by lamivudine therapy in chronic hepatitis B. J Hepatol. 2003;39(4):595-605.

18. Tan AT, et al. A longitudinal analysis of innate and adaptive immune profile during hepatic flares in chronic hepatitis B. J Hepatol. 2010;52(3):330-339.

19. Becher B, et al. High-dimensional analysis of the murine myeloid cell system. Nat Immunol. 2014;15(12):1181-1189.

20. Blackburn SD, et al. Coregulation of $\mathrm{CD}^{+} \mathrm{T}$ cell exhaustion by multiple inhibitory receptors during chronic viral infection. Nat Immunol. 2009;10(1):29-37.
21. Odorizzi PM, Pauken KE, Paley MA, Sharpe A, Wherry EJ. Genetic absence of PD-1 promotes accumulation of terminally differentiated exhausted CD8 ${ }^{+}$T cells. J Exp Med. 2015;212(7):1125-1137.

22. Shi W, et al. Hepatitis B virus subgenotyping: history, effects of recombination, misclassifications, and corrections. Infect Genet Evol. 2013;16:355-361.

23. Seto WK, et al. Treatment cessation of entecavir in Asian patients with hepatitis B e antigen negative chronic hepatitis B: a multicentre prospective study. Gut. 2015;64(4):667-672.

24. Tumeh PC, et al. PD-1 blockade induces responses by inhibiting adaptive immune resistance. Nature. 2014;515(7528):568-571.

25. Pallett LJ, et al. IL-2(high) tissue-resident T cells in the human liver: Sentinels for hepatotropic infection. J Exp Med. 2017;214(6):1567-1580.

26. Boni C, et al. Characterization of hepatitis B virus (HBV)-specific T-cell dysfunction in chronic HBV infection. J Virol. 2007;81(8):4215-4225.

27. Evans A, et al. Programmed death 1 expression during antiviral treatment of chronic hepatitis B: Impact of hepatitis B e-antigen seroconversion. Hepatology. 2008;48(3):759-769.

28. Rivino L, et al. Differential targeting of viral components by $\mathrm{CD}^{+}{ }^{+}$versus $\mathrm{CD} 8{ }^{+} \mathrm{T}$ lymphocytes in dengue virus infection. J Virol. 2013;87(5):2693-2706.

29. Wong MT, et al. Mapping the diversity of follicular helper T cells in human blood and tonsils using high-dimensional mass cytometry analysis. Cell Rep. 2015;11(11):1822-1833.

30. Finck R, et al. Normalization of mass cytometry data with bead standards. Cytometry $A$. 2013;83(5):483-494.

31. Amir el-AD, et al. viSNE enables visualization of high dimensional single-cell data and reveals phenotypic heterogeneity of leukemia. Nat Biotechnol. 2013;31(6):545-552. 